\title{
Spatial asymmetries in viewing and remembering scenes: Consequences of an attentional bias?
}

\author{
Christopher A. Dickinson ANd Helene Intraub \\ University of Delaware, Newark, Delaware
}

\begin{abstract}
Given a single fixation, memory for scenes containing salient objects near both the left and right view boundaries exhibited a rightward bias in boundary extension (Experiment 1). On each trial, a 500-msec picture and 2.5 -sec mask were followed by a boundary adjustment task. Observers extended boundaries 5\% more on the right than on the left. Might this reflect an asymmetric distribution of attention? In Experiments $2 \mathrm{~A}$ and $2 \mathrm{~B}$, free viewing of pictures revealed that first saccades were more often leftward (62\%) than rightward (38\%). In Experiment 3, 500-msec pictures were interspersed with 2.5-sec masks. A subsequent object recognition memory test revealed better memory for left-side objects. Scenes were always mirror reversed for half the observers, thus ruling out idiosyncratic scene compositions as the cause of these asymmetries. Results suggest an unexpected leftward bias of attention that selectively enhanced the representations, causing a smaller boundary extension error and better object memory on the views' left sides.
\end{abstract}

Various asymmetries have been observed in the way in which the left and right sides of space are perceived and represented. In the case of hemispatial neglect, individuals with damage to critical areas of one hemisphere (usually in the right parietal lobe) fail to report or respond to information on the contralateral side of space (e.g., Behrmann \& Geng, 2002; Heilman, Bowers, Valenstein, \& Watson, 1987; Heilman \& Valenstein, 1972; Kinsbourne, 1970; Mesulam, 1981; see, for review, Karnath, Milner, \& Vallar, 2002). In normal populations, asymmetrical processing has been observed in a number of cognitive tasks. In line bisection tasks, observers often show a bias to bisect lines to the left of center (referred to as pseudoneglect; Bowers \& Heilman, 1980; Jewell \& McCourt, 2000). A bias to begin searching on the left side of a display has been reported in conjunction search tasks (Ebersbach et al., 1996; Williams \& Reingold, 2001; Zelinsky, 1996). In the case of reading and eye movements, there is a rightward bias in the perceptual reading span for English readers (more letters can be read to the right of fixation than to the left) that reverses for readers of languages with the opposite reading direction, such as Hebrew (Pollatsek, Bolozky, Well, \& Rayner, 1981; see Rayner, 1998).

To our knowledge, neither eyetracking nor memory research has revealed any asymmetries in scene representation in normal populations. In the case of the first fixation on a scene, the lack of a bias toward the left or right is not surprising, given that observers tend to fixate the most salient objects or locations in a scene (e.g., the ones that are most visually conspicuous [Itti \& Koch, 2000,
2001; Parkhurst, Law, \& Niebur, 2002] or semantically informative [Buswell, 1935; Friedman, 1979; Henderson, Brockmole, Castelhano, \& Mack, 2007; Loftus \& Mackworth, 1978]). Unlike English prose, visual scenes do not have an inherent left-right structure. The same holds true for memory for the visual details of a scene; there is no a priori reason to expect better memory for objects and features on either the left or the right side. This is why we were very interested in an unexpected rightward bias in boundary extension for a briefly presented view of a scene (Intraub, Hoffman, Wetherhold, \& Stoehs, 2006).

Boundary extension is a constructive memory error for views of scenes in which views are remembered as being more spatially expansive than they actually were-as if the viewer had seen what would be visible just beyond the view's boundaries (Intraub \& Richardson, 1989). It is thought to reflect the fact that in the world, a scene surrounds the viewer but can never be seen all at once. Scene representation is thought to involve not only the visual sensory information observed, but also the spatial context of that view within the larger scene (e.g., Dickinson \& Intraub, 2008; Intraub \& Dickinson, 2008; see Intraub, 2007). In support of this contention, boundary extension does not occur for all types of pictures (e.g., drawings of objects on blank backgrounds), but only for those in which the background conveys a scene context (i.e., a partial view of an otherwise continuous world; Gottesman \& Intraub, 2002; Intraub, Gottesman, \& Bills, 1998). This is further supported by fMRI research (Park, Intraub, Yi, Widders, \& Chun, 2007) showing that boundary extension

C. A. Dickinson, dickinsonca@appstate.edu 
evokes selective responses in the parahippocampal place area $(P P A)$ and retrosplenial cortex $(R S C)$ - brain regions thought to be specifically related to scene layout and location (see Epstein, 2005).

Dependent measures in most boundary extension research do not speak to the issue of asymmetry because they involve an overall assessment of area (e.g., ratings of whether the test view is the same, closer-up, or more wideangle than before [Intraub \& Richardson, 1989]; a "zooming" tool that allows observers to show more or less of the picture at test [Chapman, Ropar, Mitchell, \& Ackroyd, 2005]). To date, only a few studies have allowed an assessment of boundary extension at individual boundaries.

Drawing tasks have not revealed any noticeable rightward bias in boundary extension (e.g., Intraub \& Bodamer, 1993; Intraub et al., 1998; Intraub \& Richardson, 1989), and subsequent perusal of observers' drawings has not suggested one. A boundary adjustment task, in which observers could adjust the boundaries of a window-like aperture to reveal more or less of a real 3-D scene, similarly revealed no evidence of a left-right asymmetry (Intraub, 2004). Intraub et al. (2006) used a similar type of border adjustment task to test memory for briefly presented photographs. In this case, at test, observers used the mouse to move each border inward or outward to reconstruct the remembered view. No left-right asymmetry occurred when they were required to move their eyes; however, a surprising rightward bias in the boundary error occurred when the observers were required to maintain fixation.

The purpose of Intraub et al.'s (2006) research was to determine whether a planned gaze shift would influence the amount of boundary extension beyond the to-be-fixated side of a photograph (Intraub, 2007; Intraub \& Richardson, 1989). It did not. Results indicated that boundary extension was based on the observed space, rather than on the expected, upcoming space that a new fixation would bring into view. No asymmetrical representation of space was obtained when the observers shifted their eyes to the left or right in response to a cue (the stimulus was always removed before the eyes landed). However, in the one experiment in which observers were required to maintain fixation on the center of the scene (the control experiment), the unexpected asymmetry was observed.
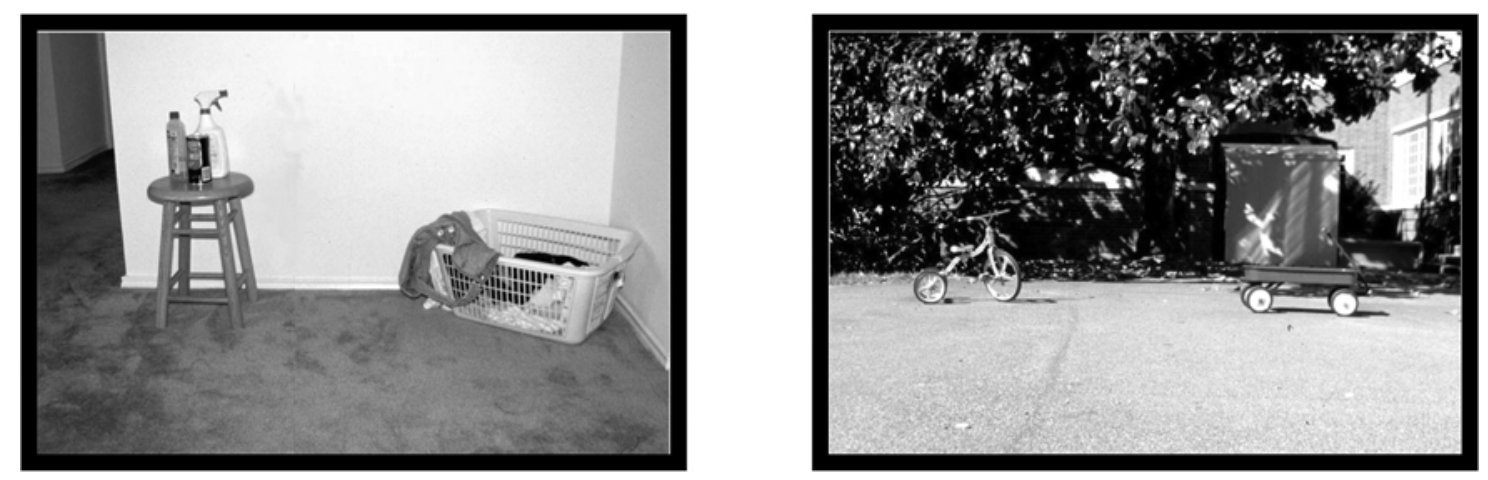

Figure 1. Examples of indoor (left) and outdoor (right) scenes used in the three experiments. Note that all the scenes were shown in color.

In these experiments, the stimuli (color photographs of scenes) had to be structured in a somewhat unusual way to ensure that the observers would always have two potential saccade targets. All views had to contain a salient object near the left and right boundaries-for example, a patio with a tricycle on one side and a child's wagon on the other (as is shown in Figure 1). On each trial of the control experiment, a picture was presented for $500 \mathrm{msec}$. During this time, the observers were required to maintain central fixation (eye movements were monitored). A 2-sec mask replaced the picture, followed by a test picture (a closer or wider view of the same stimulus). Boundary adjustment revealed a strong rightward bias; the observers remembered having seen farther beyond the right boundary (a 20\% shift outward) than beyond the left boundary (a 12\% shift outward). This surprising bias could not be attributed to left-right differences in the composition of the pictures, because pictures were mirror reversed for half of the observers, to control for this potential factor.

The rightward bias in the "shape" of the spatial representation observed in Intraub et al.'s (2006) maintain fixation condition might have been a spurious effect. However, we thought it raised an interesting possibility. Perhaps there exists a subtle bias toward the left side of space in the first fixation on a scene. When "all things are equal" in terms of the structure of the view (i.e., a salient object on each side of the picture) and the action plans of the observer (i.e., maintaining fixation), this bias can be observed. Thus, we are not interested here in the impact of a planned fixation on memory, as in Intraub et al. (2006), but in a possible bias that might exist prior to visual scanning.

\section{What Might Account for This Asymmetry in Boundary Extension?}

There are several possible explanations that have been examined in relation to observed asymmetries in visual perception and attention. One extensively investigated explanation involves functional differences between the hemispheres. The domains of language comprehension and spatial attention provide numerous examples. In lexical decision tasks, words that reach the left hemisphere first (because they were presented to the right visual field) are 
typically identified more quickly and/or more accurately than words that are presented to the right hemisphere first (e.g., Chiarello, 1985; Faust, Kravetz, \& Babkoff, 1993; Hines, 1978; Young \& Ellis, 1985). In addition, a number of studies indicate that the left and right hemispheres are involved in processing specific aspects of language (see Lindell, 2006; Schmidt, DeBuse, \& Seger, 2007). Within the domain of spatial attention, patients having unilateral lesions in the right hemisphere typically show more profound symptoms of neglect for the contralateral side of space than do patients with unilateral lesions in the left hemisphere (Bisiach, Cornacchia, Sterzi, \& Vallar, 1984; Weintraub \& Mesulam, 1987; see Bowen, McKenna, \& Tallis, 1999, for a review). Further evidence from neuroimaging studies suggests that the right hemisphere is more important than the left hemisphere in directing spatial attention (Coull \& Nobre, 1998; Gitelman et al., 1999; Nobre, Coull, Frith, \& Mesulam, 1999).

Although these, as well as other, lateral asymmetries in visual perception have been linked to specific brain regions, we suggest that testing hypotheses based on brain function would be premature at this point in our investigation. Instead, we asked whether the rightward bias in boundary extension was the result of a spatial attention bias. Recently, Intraub, Daniels, Horowitz, and Wolfe (2008) demonstrated that attentional allocation can modulate boundary extension. They found that boundary extension was greater when attention was divided in a dual-task situation, relative to when observers were attending only to the scenes. If more attention were devoted to the left side of space than to the right side during the first fixation on a scene, this might result in a smaller boundary error for the left sides of scenes.

The purpose of these experiments was to determine whether the rightward bias in boundary extension obtained after observers had maintained central fixation on briefly presented pictures could be replicated using a larger stimulus set (Experiment 1) and then, if so, to determine whether this might be caused by a leftward bias in the distribution of attention. To this end, in Experiments $2 \mathrm{~A}$ and $2 \mathrm{~B}$, we measured the direction of the first saccade away from the center in two free-viewing tasks; in Experiment 3, we tested object recognition memory for objects appearing on the right and left sides of space. If the distribution of attention is biased leftward for these balanced views of scenes (salient objects on both the left and right), an asymmetry favoring the left side of space should be apparent not only in boundary extension, but also in free viewing and in object memory as well. More specifically, we predicted a smaller boundary error for the left sides of the scenes (Experiment 1), a leftward bias in initial saccade direction under free-viewing conditions (Experiments 2A and 2B), and better memory for visual details on the left sides of scenes (Experiment 3).

\section{EXPERIMENT 1}

In Experiment 1, we attempted to replicate the rightward bias in boundary extension. On each trial, stimulus duration was $500 \mathrm{msec}$, and after a $2.5-\mathrm{sec}$ masked interval, the picture reappeared with the borders either pulled inward, revealing less of the picture, or pulled outward, revealing more. This was done so that the observers would have to move borders inward and outward equally often, thus avoiding a bias. Eyetracking allowed us to test whether the observers were following our instructions to maintain fixation and to eliminate trials on which a saccade was made. The only major difference between this experiment and the original one is that we varied the aspect ratio of the pictures so that observers could not rely on memory for the rectangular space covered by each picture. (In the original experiment, this was accomplished by varying the size of the test picture.) Minor differences included an increase in the number of trials to 30 and an increase in the retention interval from 2 to $2.5 \mathrm{sec}$.

\section{Method}

Participants. A total of 24 University of Delaware undergraduates, fulfilling a requirement for an introductory psychology course, participated in the experiment. All reported having normal or corrected-to-normal vision and normal color vision.

Apparatus. All the stimuli were presented on a 21-in. flat-screen CRT monitor in 32-bit color at a resolution of 1,024 $\times 768$ pixels and a refresh rate of $120 \mathrm{~Hz}$ that was driven by a video card with $128 \mathrm{MB}$ of video memory. Stimulus presentation was controlled by a Pentium-based PC running Microsoft Windows XP. The software was based on a template program supplied by SR Research Inc., written in C, that used Simple DirectMedia Layer (SDL) Version 1.2.9. We used an EyeLink II video-based eyetracking system to collect eye movement data (SR Research Ltd.). Eye position was sampled at $500 \mathrm{~Hz}$, the system's spatial resolution was estimated to be less than $0.4^{\circ}$, and head position and viewing distance of $72 \mathrm{~cm}$ were fixed with a chinrest.

Stimuli. The stimuli consisted of a set of 41 digitized color photographs of indoor and outdoor scenes with a salient object or object cluster on both the left and right sides. Figure 1 shows representative indoor and outdoor pictures. Eleven of the pictures were used for practice trials; the remaining 30 were used for experimental trials ( 12 of these were from the set of pictures used by Intraub et al., 2006). The pictures were more wide-angle than the stimulus views shown during presentation so that, during the test, the observers would have the option of adjusting the boundaries to show more or less of the picture than before. On average, stimulus views subtended $14.8^{\circ} \times 9.9^{\circ}$ of visual angle (widths ranged from $11.2^{\circ}$ to $16.4^{\circ}$; heights ranged from $7.0^{\circ}$ to $11.7^{\circ}$ ). Figure 2 shows a representative picture, along with its stimulus view and initial test views. Initial test views that showed less of the picture than the stimulus view (referred to as small-aperture trials) subtended $1^{\circ} \times 1^{\circ}$ of visual angle; those that showed more of the picture than the stimulus view (referred to as large-aperture trials) subtended $22.8^{\circ} \times 14.6^{\circ}$.

Design and Procedure. A depiction of a trial is shown in Figure 3. The observers self-initiated each trial while fixating on a central fixation cross (this also served as drift correction for the eyetracker). After $500 \mathrm{msec}$, the stimulus view of the picture was displayed for a total of $500 \mathrm{msec}$. A red circle appeared around the fixation cross $250 \mathrm{msec}$ after the picture's onset and remained visible for $50 \mathrm{msec}$ (to remind the observers to maintain central fixation). A full-screen noise mask was shown immediately after the stimulus for $2,000 \mathrm{msec}$, followed by a 500 -msec blank screen. The observers were instructed to maintain central fixation during stimulus presentation and to try to remember the pictures in as much detail as possible. They were told that the background and layout of the objects were as important to remember as the objects themselves and that boundary memory would be tested on each trial.

The blank screen was followed immediately by the memory test. Here, the observer's task was to re-create the initial stimulus view as 
A

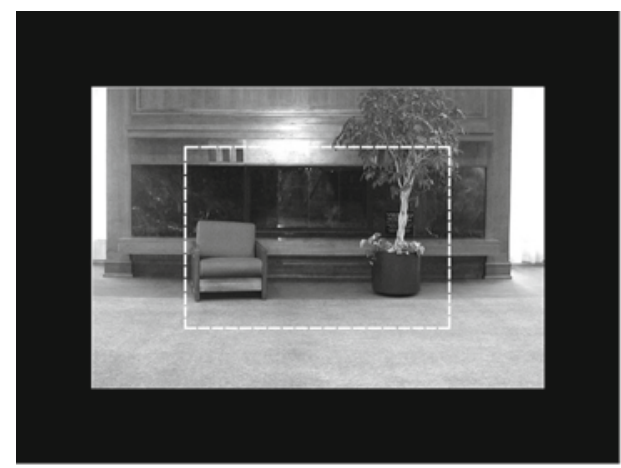

C

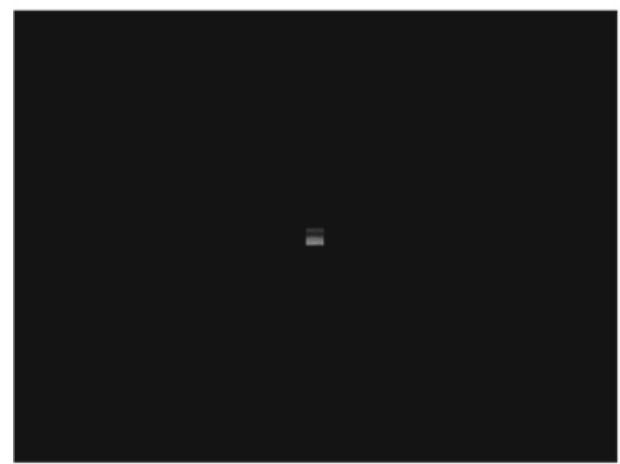

$\mathbf{E}$

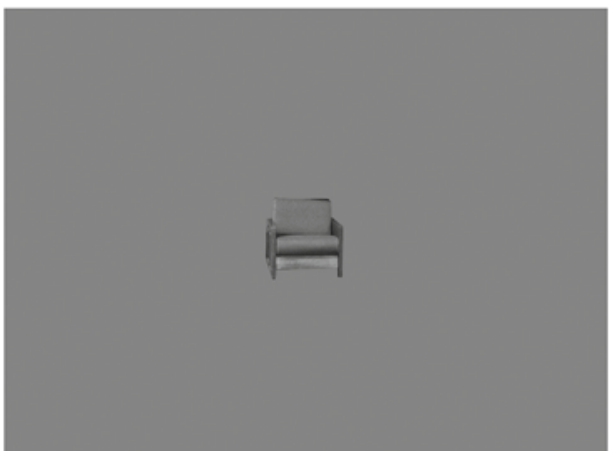

B

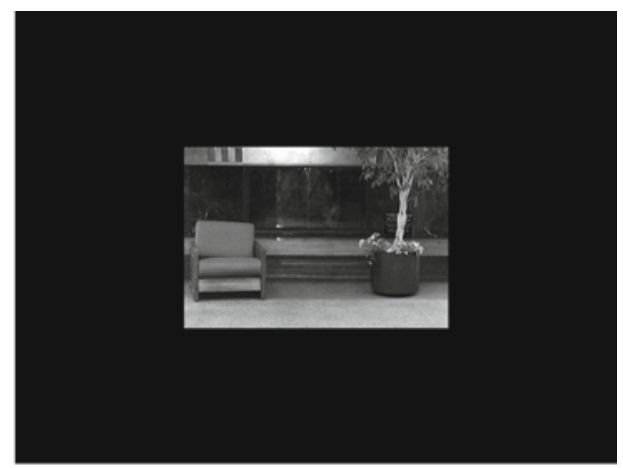

D

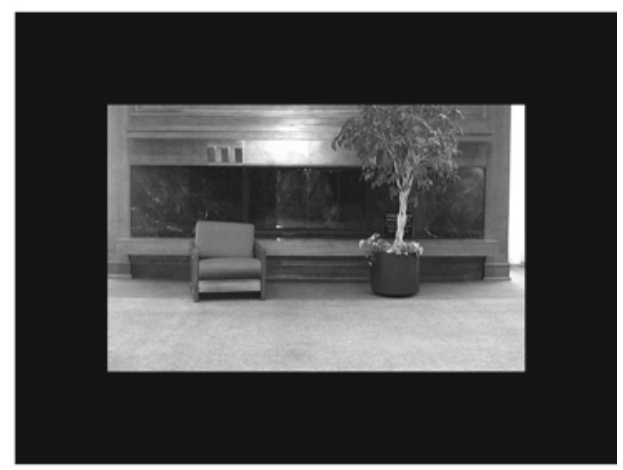

$\mathbf{F}$

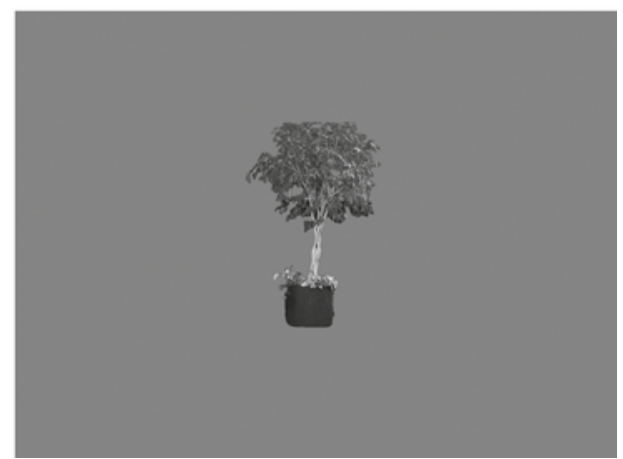

Figure 2. A representative scene and its stimulus view, initial test views, and cut-out objects used for the Experiment 3 object recognition test. (A) Wide-angle view showing stimulus view (indicated by dotted lines). (B) Stimulus view with black borders. (C) Small-aperture initial test view. (D) Large-aperture initial test view. (E) The left-side test object. (F) The right-side test object.

accurately as possible by using the mouse to move each black border outward or inward to reveal more or less of the picture. The program created the black borders by superimposing black rectangles on the top, bottom, left, and right portions of the test picture. The observers then indicated how confident they were about their border placement by clicking sure (3), pretty sure (2), not sure (1), or don't remember that picture $(0)$. This was followed by eye movement feedback that indicated whether central fixation was maintained during stimulus presentation. The next fixation cross was presented immediately after the feedback.

On half of the experimental trials, the initial test view revealed only a small central portion of the picture (referred to as small-aperture trials); on the other half, the initial test view revealed much more of the picture than was initially shown during stimulus presentation (referred to as large-aperture trials), as is shown in Figures $2 \mathrm{C}$ and $2 \mathrm{D}$. At the start of the session, the observers were given 20 practice trials to practice maintaining central fixation (each practice picture was shown twice: in its normal orientation and mirror reversed). The observers were then given 1 practice trial with the boundary adjustment task. During practice trials, the eye movement feedback followed immediately after the blank interval. Half of the observers were shown the photographs in their normal orientations, and the other half were shown each photograph's mirror reversal to control for any effects of left-right composition of the photographs. In addition, initial test view (small vs. large aperture) was counterbalanced across observers.

Trials on which the observers reported not recognizing the test picture $(0.4 \%)$ and trials on which the observers failed to maintain 


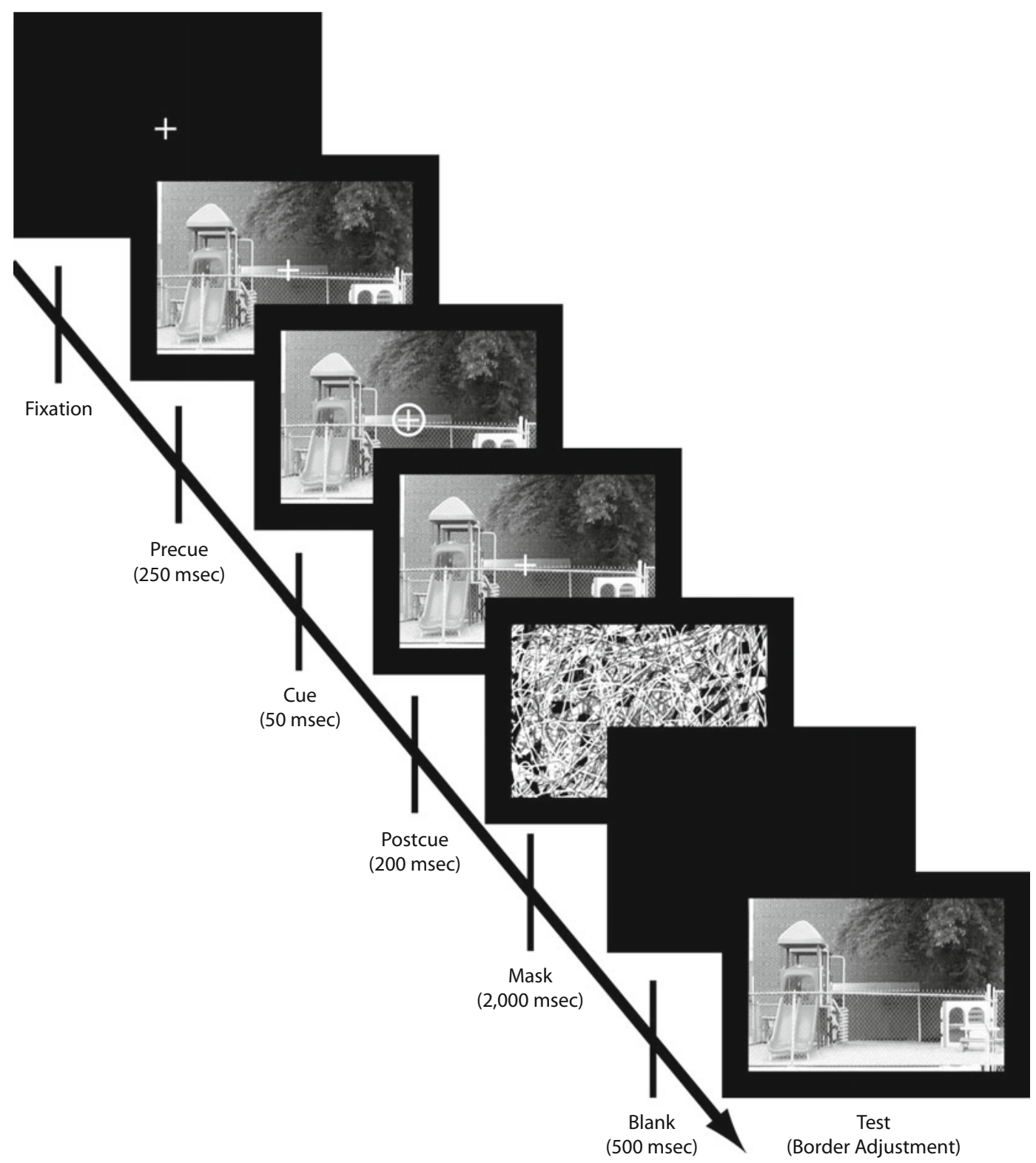

Figure 3. A depiction of a trial in Experiment 1. Note that the stimuli did not fill the screen and that the initial test view was never identical to the stimulus view.

fixation during stimulus presentation $(8.3 \%)$ were excluded from all the analyses.

Measurement of border adjustments. Percent change in border position was determined relative to the center of the test picture. For example, if the distance from the picture's center to the left stimulus border was 100 pixels and the distance from the picture's center to the final adjusted position of the left test border was 110 pixels, this would be reflected as a $10 \%$ increase in the amount of space shown on the left side of the picture. Boundary extension for the overall expanse of the pictures is expressed as the percent difference in area of the adjusted test pictures, relative to the area of the stimulus views.

\section{Results and Discussion}

Boundary extension occurred; on average, the observers revealed $16 \%$ more of the scene than had been visible in the stimulus. This was significantly greater than a mean of $0 \%$ change $[t(23)=5.04, p<.001]$. The critical change in border placement on the left and right sides is shown in Figure 4 , with the $95 \%$ confidence intervals around each mean. As is shown in Figure 4, a significant rightward bias in boundary extension was found: The observers revealed significantly more of the right side of the scenes than the left side both for small-aperture trials [right vs. left, $8.5 \%$ vs. $5.5 \% ; t(23)=2.3, p<.05]$ and for large-aperture trials [right vs. left, $13.8 \%$ vs. $6.6 \% ; t(23)=4.1, p<.001$ ] In addition, the observers were fairly confident in their border adjustments; mean confidence ratings for smallaperture trials (1.89) and large-aperture trials (1.97) did not differ significantly $[t(23)=1.54$, n.s. $]$.

As in previous studies, boundary extension occurred whether the observers had to move the borders inward 


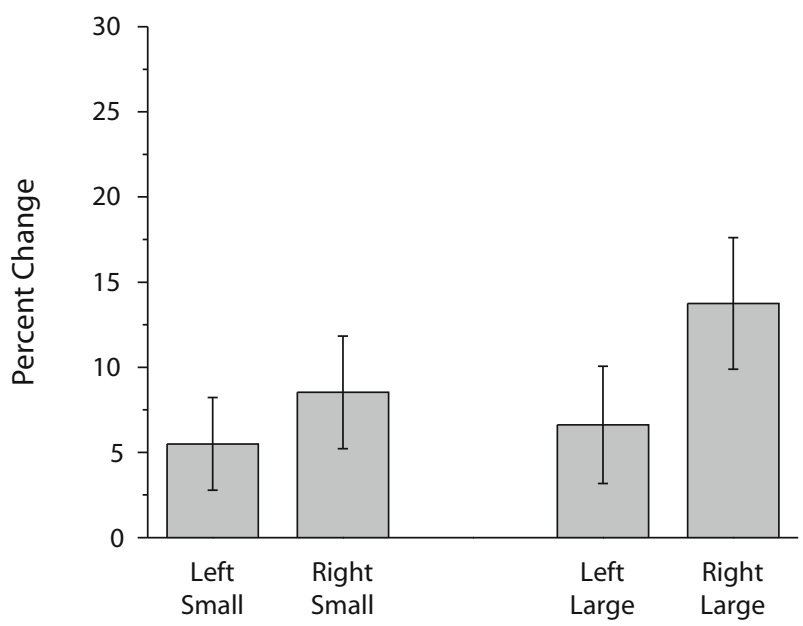

Figure 4. Mean percent change in left and right border positions for small-test-aperture trials and large-test-aperture trials in Experiment 1. All error bars indicate $95 \%$ confidence intervals. Means significantly greater than zero indicate boundary extension.

[mean increase in area, $11 \% ; t(23)=3.64, p<.005]$ or outward [mean increase in area, $22 \% ; t(23)=5.21, p<$ $.001]$ when they re-created their remembered views. As in other experiments, however, ultimate boundary placement yielded more boundary extension when the initial test view showed more than the stimulus view than vice versa $[t(23)=3.41, p<.005$; Chapman et al., 2005; Intraub et al., 2006]. This difference is thought to be due either to an anchoring effect or to a weak representation of extended space being activated when the wider angle test views (large-aperture trials) are seen.

It should be noted that factors affecting individual sides of a view in boundary extension are just beginning to be explored. As was described earlier, no asymmetries were previously noted in drawings (e.g., Intraub \& Richardson, 1989) or in border adjustment tasks conducted in real 3-D space (Intraub, 2004). In addition, a left versus right asymmetry did not occur in the eye movement conditions in Intraub et al. (2006) when observers were cued to fixate an object on the left or right, $250 \mathrm{msec}$ into a $500-\mathrm{msec}$ exposure. The asymmetry that occurred in their control experiment (when observers simply maintained fixation), and that we have now replicated, was unexpected.

Why might this asymmetry in boundary extension have occurred? As was suggested earlier, one possibility is that there is a subtle bias in the distribution of spatial attention toward the left side of space during the first fixation on a scene, prior to the onset of a focal attention shift. This bias may have enhanced detail retention on the left side of the picture, which served to attenuate the boundary extension error on that side, as compared with the right side. "All things being equal" (salient objects on both sides of the picture; observer maintains fixation on the center), this bias was observed. This qualification appears to be a very important one because, when focal attention was manipulated via a directional cue in Intraub et al.'s (2006) eye movement conditions, the region toward which attention was shifted did not show a reduction in boundary extension.

This suggests that the leftward bias of attention is a subtle one. In Intraub et al.'s (2006) eye movement conditions, the demands of the task (e.g., interpreting the cue, selecting the object, launching the saccade) may not have been conducive to selecting and retaining the types of relational details that might help attenuate boundary extension. Alternately, it may be that the task of maintaining attention versus making a saccade in a response to a directional cue might draw on different aspects of attention (e.g., distributed in the case of maintaining fixation and attempting to memorize the scene vs. focal in the case of launching a saccade to fixate a specific object). Distributed attention (while maintaining fixation) might be more conducive to encoding relational aspects of the stimulus that serve to attenuate boundary extension in memory.

\section{EXPERIMENTS 2A AND 2B}

Perhaps the rightward bias in boundary extension reflects a leftward bias in the initial distribution of attention during observers' initial fixations on pictures. On the basis of the obligatory coupling between covert attention shifts and saccades (Deubel \& Schneider, 1996; Hoffman \& Subramaniam, 1995; Kowler, Anderson, Dosher, \& Blaser, 1995; Shepherd, Findlay, \& Hockey, 1986), it is possible that the eyes might be "drawn" to the side of space to which attention is biased. In Experiments 2A and 2B, we therefore asked whether we would find a corresponding leftward bias in the direction of observers' initial saccades when they viewed the pictures. To explore this question, we presented the same pictures in the same order as in Experiment 1, allowed free viewing, and monitored eye movements.

The critical question was whether there would be an overall leftward bias in the direction of the first saccade or whether its direction would be determined by idiosyncrasies of individual pictures. Again, pictures were mirror reversed for half of the observers in order to ensure that any biases in initial saccade direction were not caused by idiosyncrasies of the pictures. If the observers were biased to initially attend to the left side of the pictures, we should observe a corresponding bias in initial saccade direction. In Experiment 2A, each picture was presented for $500 \mathrm{msec}$, as in the previous experiment. In Experiment $2 \mathrm{~B}$, each picture was presented for $10 \mathrm{sec}$ to determine whether a bias in initial saccade direction might be evident under conditions in which the observers were not under demanding time pressure, more like normal free viewing.

To avoid biasing the observers' gaze, the memory test was deferred until the end of the sequence. We simply sought to determine where people looked first. In Experiment $2 \mathrm{~A}$, we gave the observers a brief picture recognition test to follow through on our instruction that memory for the pictures would be tested. In Experiment 2B, we decided to include a boundary memory test similar to that 
in Experiment 1 to determine whether, as in Intraub et al. (2006), the rightward bias would be eliminated if the observers moved their eyes during presentation. We tested a subset of the pictures, chosen by selecting the 11 pictures that had revealed the greatest rightward bias in Experiment 1 . To make study conditions as comparable as possible among the three experiments (at least within the first fixation), the observers were given the same instructions for studying the pictures as in the previous experiment.

\section{Method}

Participants. A total of 24 University of Delaware undergraduates from the same population as that in Experiment 1 participated in Experiment 2A; 29 undergraduates from the same population participated in Experiment 2B. None had participated in the previous experiment.

Apparatus and Stimuli. The apparatus was the same as in Experiment 1 . The stimuli were selected from the same pool of pictures as in the previous experiment. For pictures shown in the previous experiment, stimulus views were identical to the views shown in that experiment. For the remaining pictures, stimulus views were within the same size range. There were 44 stimuli in Experiment 2A and 34 stimuli in 2B. Four additional pictures were used for the practice trials.

Design and Procedure. The observers self-initiated each picture in the presentation sequence while fixating on a central fixation cross. After $500 \mathrm{msec}$, the stimulus view of the picture was displayed for a total of $500 \mathrm{msec}$ in Experiment 2A and for $10 \mathrm{sec}$ in Experiment 2B. In both cases, the stimulus picture was followed by a noise mask for 2,000 msec and a 500-msec blank screen. The next fixation cross appeared at the end of the blank interval. As in Experiment 1, the observers were instructed to study each picture for a subsequent memory test and were told that the background and layout of the objects were as important to remember as the objects themselves. In Experiment 2A, the observers viewed 40 pictures, which included the 30 experimental pictures from Experiment 1 and 10 additional pictures from our stimulus set. In Experiment 2B, the observers viewed only the 30 experimental pictures from Experiment 1 in order to allow us to examine whether any biases in initial saccade direction would be found in the identical set of pictures that yielded the rightward bias in boundary extension found in the previous experiment.

After the observers had viewed the presentation sequence, they were given the instructions for the memory test. In Experiment 2A, the observers were given a brief picture recognition test, simply to follow through on the instruction that memory for the pictures would be tested. In Experiment 2B, the observers were given a border adjustment test. They were tested on the 11 pictures that had yielded the largest rightward bias in boundary extension in Experiment 1, and after each border adjustment, they indicated their confidence with the same four-choice scale as that in Experiment 1. As in the previous experiment, photograph orientation (normal vs. mirror reversed) and initial stimulus view (large aperture vs. small aperture) were counterbalanced across observers. At the start of the session, the observers in each experiment were given four practice trials.

Data inclusion criteria. Data from several studies suggest that at least $150 \mathrm{msec}$ is required to program and initiate a saccade (see Rayner, 1998). Thus, it would be unlikely that a saccade initiated sooner than $150 \mathrm{msec}$ after stimulus onset could be influenced by pictorial information. We therefore analyzed eye movement data from only trials on which the initial saccade was initiated at least 150 msec after stimulus onset.

In Experiment 2A, we included all 24 observers in the initial saccade analyses, because of the large number of pictures; however, we excluded $24 \%$ of the initial saccades on the basis of the saccade latency criterion (with individual observers having between $43 \%$ and 95\% valid trials). In Experiment 2B, however, because of the smaller number of pictures in the presentation sequence, we required that at least $70 \%$ of an observer's trials meet the saccade latency criterion for his or her data to be included in the analyses. This resulted in the exclusion of the data from 5 of the initial observers; their data were replaced with data from 5 additional observers ( 1 of whom had fewer than 50\% valid trials and whose data were not included in any analyses). The saccade latency criterion resulted in the exclusion of $7.5 \%$ of initial saccades from the remaining 23 observers.

\section{Results and Discussion}

In general, the observers showed a striking leftward bias in initial saccade direction in both Experiment 2A [left vs. right, $63 \%$ vs. $37 \% ; t(23)=4.45, p<.001$ ] and Experiment $2 \mathrm{~B}$ [left vs. right, $62 \%$ vs. $38 \%$; $t(22)=$ $3.57, p<.005]$. In Experiment $2 \mathrm{~A}$, initial saccade latencies were shorter for leftward saccades than for rightward saccades [left vs. right, 240 vs. $278 \mathrm{msec} ; t(23)=4.35$, $p<.001]$, but initial saccade amplitude did not differ as a function of initial saccade direction (left vs. right, $3.21^{\circ}$ vs. $3.24^{\circ} ; t<1$ ). In Experiment 2B, there were no significant differences in initial saccade latency [left vs. right, 281 vs. $330 \mathrm{msec} ; t(22)=1.45$, n.s.] or amplitude (left vs. right, $3.55^{\circ}$ vs. $3.37^{\circ} ; t<1$ ) as a function of initial saccade direction. The leftward bias in initial saccade direction was not due to picture idiosyncrasies, because the same pictures were presented mirror reversed to half the observers, and in both orientations, the leftward bias for the initial saccade occurred in both Experiment 2A (both orientations, $63 \%$ leftward) and Experiment 2B (original orientations, $60 \%$ leftward; mirror-reversed orientations, $63 \%$ leftward).

The boundary adjustment test in Experiment $2 \mathrm{~B}$ yielded no difference in boundary extension on the left and right sides of the pictures. The mean percent change in border position for the left and right borders and the $95 \%$ confidence intervals around each mean are shown in Figure 5. In addition, the observers were fairly confident in their border adjustments; mean confidence ratings for smallaperture trials (2.10) and large-aperture trials (2.06) did not differ significantly $(t<1)$. Thus, the rightward bias in boundary extension observed in memory for these pictures in Experiment 1 did not occur when the observers could shift fixation (and attention) over the course of several seconds during presentation and memory was tested at the end of the sequence. As in Intraub et al. (2006), the rightward bias in boundary extension occurred only when the observer maintained fixation on a briefly presented picture, suggesting an underlying bias in the distribution of attention prior to the onset of visual scanning. Taken together with the single-saccade conditions in Intraub et al. (2006), these data suggest that (1) the time frame for such a biased representation of a view is very brief (it may be available only prior to the onset of a focal attention shift) and (2) a focal attention shift eliminates the bias, even when the shift is leftward (which might be expected to at least maintain the bias).

Pursuant to our hypothesis, however, the present experiment clearly revealed a leftward bias in initial saccade direction. This suggests that when pictures were first presented, the observers' attention was distributed unequally, 


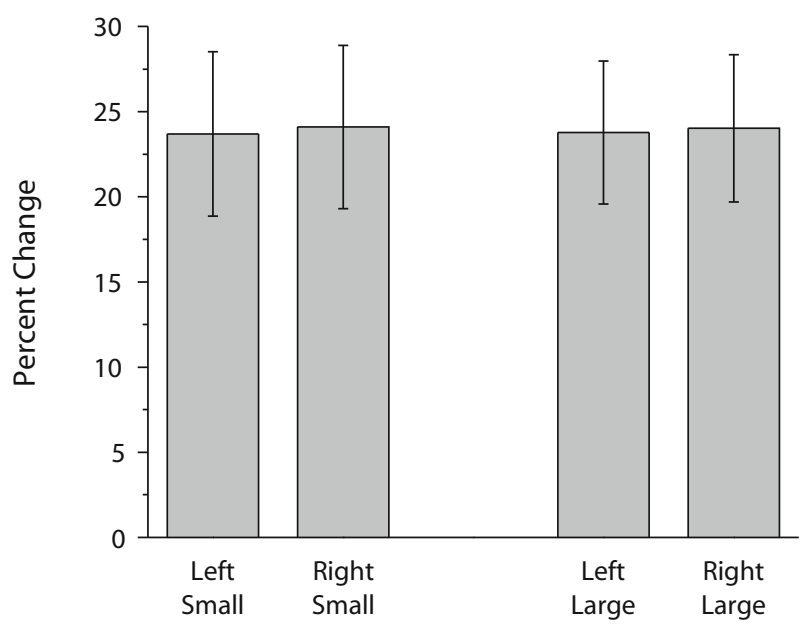

Figure 5. Mean percent change in left and right border positions for small-test-aperture trials and large-test-aperture trials in Experiment 2B. All error bars indicate $95 \%$ confidence intervals. Means significantly greater than zero indicate boundary extension.

with a bias to focus more attention on the left side of space. As was mentioned earlier, a similar bias in initial saccade direction has also been found in challenging visual search tasks (Williams \& Reingold, 2001; Zelinsky, 1996). This leftward bias in initial saccade direction is consistent with an attentional modulation explanation of the rightward bias in boundary extension observed in Experiment 1. Greater focus on the left might result in a slightly more veridical spatial representation. In the next experiment, we examined whether we could find converging evidence for greater attention to the left side of space that would influence scene memory.

\section{EXPERIMENT 3}

The eyetracking data from Experiments $2 \mathrm{~A}$ and $2 \mathrm{~B}$ indicated that, initially, the observers were biased to attend to the left sides of the pictures, even though there was a salient object on each side and, across observers, the same pictures were mirror reversed. This provides support for the hypothesis that the rightward bias in boundary extension observed in Experiment 1 may have been the result of a leftward bias in the distribution of attention that limited the amount of distortion on that side. In Experiment 3 , we sought to find converging evidence for this hypothesis, again using briefly presented pictures (as in Experiment 1), but using a different measure of attention: recognition memory for objects on the left versus right side of the pictures.

It is clear that memory for objects in a scene depends not only on attention, but also on familiarity and other factors that will vary across observers. Our rationale was that if attention tends to be biased to the left side of space for these stimuli, we might expect to see somewhat better recognition performance for objects that appeared on that side of space. Object recognition tests have shown that fixated (and thus attended) objects are remembered better than nonfixated objects in a scene (Henderson \& Hollingworth, 1999; Hollingworth \& Henderson, 2002; Irwin \& Zelinsky, 2002). A null effect would be difficult to interpret, but a difference favoring the left would provide converging evidence for a leftward bias of attention.

\section{Method}

Participants. A total of 26 University of Delaware undergraduates from the same population as that in the previous experiments participated in the experiment. We replaced 2 observers whose performance on the memory test was at the level of chance. None had participated in any of the previous experiments.

Apparatus and Stimuli. The apparatus and stimuli were the same as those in Experiments 1 and 2, except for the addition of 10 new pictures that were added to the series to increase set size to 40 for the purpose of minimizing the likelihood of a ceiling effect in performance on the memory test. They were presented as part of the first and last five trials, and memory was not tested for these pictures.

Design and Procedure. The observers self-initiated each picture in the sequence while fixating on a central fixation cross. After $500 \mathrm{msec}$, the picture was shown for $500 \mathrm{msec}$ (with the fixation cross still visible), followed by a full-screen noise mask for $2,000 \mathrm{msec}$ and a $500-\mathrm{msec}$ blank screen. The next fixation cross appeared at the end of the blank interval. The observers were instructed to maintain central fixation while viewing the pictures and were told to try to remember each one for a subsequent memory test. As in the previous experiments, they were told that the background and the layout of the objects were as important to remember as the objects themselves. The observers were not informed about the type of memory test until all 40 pictures had been shown.

Test items were created as follows. A subset of 20 pictures was selected to be tested, and the left-side and right-side objects from each were copied onto a homogeneous gray background, as is illustrated in Figures 2E and 2F. In the stimulus views, one object was slightly cropped by a boundary in seven scenes, and both were slightly cropped in nine other scenes. Because cropped objects look odd when taken out of their scene context, test items were taken from the wide-angle version of each picture (e.g., Figure 2A). This was explained to the observers in the memory test instructions. Distractor objects were created in the same manner and were taken from a separate set of digitized color photographs of indoor and outdoor scenes. None of the distractor objects were visually similar to any of the target objects, although in eight cases, they shared the same basic-level category (e.g., a blue plastic recycling bin with a lid vs. a gray plastic trash container).

The test sequence was constructed as follows. One of the objects from each picture was presented in the first half of the test sequence, intermixed with 10 distractors. Half were objects from the left side and half from the right side of the pictures. The other object from each picture was presented in the second half of the test sequence, intermixed with the remaining 10 distractor objects. The order of objects in each half was pseudorandom, with two constraints: (1) that no more than two left-side targets, right-side targets, or distractors could appear in a row, and (2) that two objects from the same picture were never presented in the same serial position. The memory test was a 60 -item yes-no object recognition test in which test objects were presented individually in the center of the screen and the observers indicated whether they remembered seeing the object by clicking yes or no with the mouse. The observers indicated their confidence in their decisions after each recognition response by clicking sure (3), pretty sure (2), or not sure (1).

The object tested first (left vs. right) and stimulus orientation (normal vs. mirror reversed) were fully crossed and counterbalanced across observers. At the start of the session, the observers were given four practice trials. Trials on which the observers did not maintain fixation during stimulus presentation (16\%) were not included in any analyses. 


\section{Results and Discussion}

We computed $A^{\prime}$ values for each participant as a composite index of recognition memory, with the false alarm rate for both left-side and right-side targets based on the same set of distractors. We used $A^{\prime}$ because it is a nonparametric analogue of $d^{\prime}$ that is more appropriate for yes-no recognition tests (Pollack, 1970; Pollack \& Norman, 1964; Snodgrass, Volvovitz, \& Walfish, 1972). Object recognition memory was slightly but significantly superior for objects that had appeared on the left side of pictures than for objects that had appeared on the right side: left-side and right-side $A^{\prime}$ values were .72 and .67 , respectively $[t(23)=2.42, p<.05]$. The same pattern was found for the hit rates: left-side and right-side hit rates were .56 and .47 , respectively $[t(23)=2.78, p<.05]$ (the false alarm rate was .27). This suggests that the observers' attention was distributed unequally across the pictures, with more attention devoted to the left side of space. In addition, the observers were fairly confident in their responses: Mean confidence judgments for correct responses to left-side objects (2.41) and right-side objects (2.36) did not differ $[t(23)=1.18$, n.s. $]$. The observers were more confident in correct responses (2.38) than in false alarms (2.12), however $[t(23)=3.35, p<.005]$.

\section{GENERAL DISCUSSION}

Three experiments provided converging evidence that attention is not evenly distributed across a scene during the first fixation. With salient objects on each side of our briefly presented photographs and fixation focused on the center, we found that both boundary memory (Experiment 1 ) and object recognition memory (Experiment 3 ) were better for the left sides of scenes than for the right sides. In Experiments 2A and 2B, under free-viewing conditions, the direction of the first saccade (away from the center of the picture) was leftward on fully $62 \%$ and $63 \%$ of the trials, respectively. These asymmetries cannot be attributed to idiosyncratic picture composition, because in each experiment for half the observers, the images were mirror reversed.

In Experiment 1, the observers adjusted the borders of a test picture seconds after viewing each stimulus. A rightward bias was observed, thus replicating the unexpected asymmetry reported in Intraub et al. (2006). In both experiments, this asymmetry occurred only when the observers maintained fixation on the center of a briefly presented picture. On the basis of recent research showing that boundary extension is greater when attention is divided during encoding than when it is focused (Intraub et al., 2008), we hypothesized that better boundary memory on the left might signify a leftward bias in the distribution of attention across the scene prior to visual scanning. The results of Experiments 2 and 3 supported this hypothesis.

Because spatial attention precedes a saccade (Hoffman \& Subramaniam, 1995; Kowler et al., 1995), in Experiments $2 \mathrm{~A}$ and $2 \mathrm{~B}$, we sought to determine whether, under free-viewing conditions, the observers would exhibit a bias to initially look to the left. In both cases, about two thirds of first saccades were made to the left sides of the pictures. This pattern occurred whether the scenes were presented briefly (Experiment 2A) or for relatively long durations (Experiment 2B). In Experiment 3, we reasoned that if attention were biased leftward, improving boundary memory on the left and biasing the direction of the first saccade to the left, we should also observe better memory for objects that had appeared on the left side of the scene. An object recognition memory test confirmed this prediction. Although many factors can influence why a given observer will remember a given object, there was a slight advantage in object memory for those objects that had appeared on the left sides of the pictures.

We believe that it is important not to overstate the impact of this bias. It appears to be quite fleeting. When observers did not maintain central fixation on a briefly presented scene and, instead, launched a single saccade (Intraub et al., 2006) or made multiple saccades while viewing the scenes for multisecond durations (Experiment 2B), no evidence of the asymmetry was observed. Although evident in the experiments reported here, in which salient objects appeared on both the left and right sides of the scene, we would expect that it would be readily swamped by more idiosyncratic organizations of salient objects (see, e.g., Henderson et al., 2007). The results of Experiments 2 and 3 were as unexpected as the original boundary extension asymmetry that we replicated in Experiment 1. There is no a priori theoretical reason to predict a leftward bias when observers view balanced pictures such as these. However, the discovery of this bias has provided some new insights, as well as raised new questions, about scene representation.

A leftward bias in the distribution of attention provides a parsimonious explanation for the three asymmetries we report. It appears to affect memory for both bottom-up information (physical details about objects present in the stimulus) and top-down information (memory for unseen regions beyond the boundaries of the view), as well as influencing the direction of the observers' first gaze shift. Why the bias would occur remains an open question. It is clear, however, that researchers have been wrestling with the question of lateral biases for quite some time (e.g., Bryden \& Rainey, 1963; Heilman \& Valenstein, 1985; Mishkin \& Forgays, 1952). Perhaps the bias is related to hemispheric specialization, which has been implicated in lateral asymmetries in global versus local processing (Delis, Robertson, \& Efron, 1986; Martin, 1979; Volberg \& Hübner, 2004) and language processing (Babkoff, Faust, \& Lavidor, 1997; Ellis, Young, \& Anderson, 1988; Leiber, 1976), among others.

Alternatively, the bias may be related to reading direction (Fecteau \& Enns, 2005; Morikawa \& McBeath, 1992; Pollatsek et al., 1981; Spalek \& Hammad, 2005). This suggestion may seem counterintuitive, given that two of the leftward biases we observed (a smaller boundary error in Experiment 1, better memory for objects in Experiment 3) occurred when saccades were not allowed and that reading involves the active execution of saccades. Consistent with this suggestion, however, Spalek and Hammad found that when observers were instructed to maintain fixation, 
the magnitude of inhibition of return was greater in the left visual field for observers who read from left to right but was greater in the right visual field for observers who read from right to left. This possibility raises the intriguing question of whether individuals whose languages read from right to left would show biases in the directions opposite the biases observed in the present experiments.

In considering why this subtle asymmetry might occur, it is important to recognize that another leftward spatial bias has been reported. This phenomenon is known as pseudoneglect (Bowers \& Heilman, 1980; Jewell \& McCourt, 2000). When observers are shown a stimulus that extends to the left and right visual fields, typically, judgments of the relative magnitude of a given property on the left and right sides tend to be biased toward the left. For example, observers typically bisect horizontal lines to the left of center (Hausmann, Corballis, \& Fabri, 2003; McCourt, 2001; Nicholls \& Roberts, 2002; Varnava \& Halligan, 2007). This bias has also been found for judgments of luminance (e.g., the grayscales task; Mattingley et al., 2004; Nicholls \& Roberts, 2002), size (Charles, Sahraie, \& McGeorge, 2007; Nicholls, Bradshaw, \& Mattingley, 1999), and numerosity (Luh, Rueckert, \& Levy, 1991; Nicholls et al., 1999). In addition, similar leftward asymmetries for mental representations of number lines (Göbel, Calabria, Farnè, \& Rossetti, 2006) and alphabet lines (Nicholls \& Loftus, 2007), both of which have a spatial component, have been found.

The results of several studies support a leftward attentional bias as the mechanism underlying pseudoneglect (Bultitude \& Aimola Davies, 2006; McCourt, Garlinghouse, \& Reuter-Lorenz, 2005; McCourt \& Jewell, 1999; Nicholls \& Roberts, 2002). A bias to allocate more attention to the left side of a stimulus would result in the information on that side of space being weighted more heavily in judgments of magnitude, leading to a leftward bias in magnitude estimation. To this end, McCourt and Jewell have shown that several variables that modulate both the magnitude and direction of line bisection in patients with hemispatial neglect also modulate the magnitude and direction of pseudoneglect in non-brain-injured observers in a similar manner. In addition, a recent fMRI study by Siman-Tov et al. (2007) proposed a model of visual attentional asymmetry that can account for both left neglect seen in patients with right parietal damage and pseudoneglect seen in non-brain-injured patients.

Our research suggests that further exploration of a potential leftward bias in how the brain processes scenes is warranted. Clearly, it is a subtle effect, and without a highly contrived stimulus set (i.e., salient objects on both sides of each picture), it would likely not have been observed. Yet it is that very control that makes the bias so intriguing. Irrespective of what the cause of this asymmetry ultimately turns out to be, the present research underscores the fact that visual scene perception involves more than memory for the visually perceived information. It also includes the spatial context within which a given view is understood. The research suggests that both are part of the same representation, and whether the original source of the informa- tion is bottom up (as in the case of object recognition) or top down (as in the case of boundary extension), attention serves to enhance the veridicality of what we remember.

\section{AUTHOR NOTE}

This research was supported by NIMH Grant RO1MH54688 (to H.I.). We thank Robert D. Gordon and Kristin O. Michod for their helpful comments on the manuscript, and we thank Kari Schweifel and Mattie Wilson for their valuable assistance in preparing stimuli. Correspondence concerning this article should be addressed to C. A. Dickinson, Department of Psychology, Appalachian State University, P.O. Box 32109, Boone, NC 28608, or H. Intraub, Department of Psychology, University of Delaware, Newark, DE 19716 (e-mail: dickinsonca@appstate.edu or intraub@udel.edu).

\section{REFERENCES}

Babkoff, H., Faust, M., \& Lavidor, M. (1997). Lexical decision, visual hemifield and angle of orientation. Neuropsychologia, 35, 487-495.

Behrmann, M., \& GenG, J. J. (2002). What is "left" when all is said and done? Spatial coding and hemispatial neglect. In H. O. Karnath, A. D. Milner, \& G. Vallar (Eds.), The cognitive and neural bases of spatial neglect (pp. 85-100). New York: Oxford University Press.

Bisiach, E., Cornacchia, L., Sterzi, R., \& Vallar, G. (1984). Disorders of perceived auditory lateralization after lesions of the right hemisphere. Brain, 107, 37-52.

Bowen, A., McKenna, K., \& Tallis, R. C. (1999). Reasons for variability in the reported rate of occurrence of unilateral spatial neglect after stroke. Stroke, 30, 1196-1202.

Bowers, D., \& Heilman, K. M. (1980). Pseudoneglect: Effects of hemispace on a tactile line bisection task. Neuropsychologia, 18, 491-498.

Bryden, M. P., \& Rainey, C. A. (1963). Left-right differences in tachistoscopic recognition. Journal of Experimental Psychology, 66, 568-571.

Bultitude, J. H., \& Aimola Davies, A. M. (2006). Putting attention on the line: Investigating the activation-orientation hypothesis of pseudoneglect. Neuropsychologia, 44, 1849-1858.

Buswell, G. T. (1935). How people look at pictures: A study of the psychology of perception in art. Chicago: University of Chicago Press.

Chapman, P., Ropar, D., Mitchell, P., \& Ackroyd, K. (2005). Understanding boundary extension: Normalization and extension errors in picture memory among adults and boys with and without Asperger's syndrome. Visual Cognition, 12, 1265-1290.

Charles, J., Sahraie, A., \& McGeorge, P. (2007). Hemispatial asymmetries in judgment of stimulus size. Perception \& Psychophysics, 69, 687-698.

Chiarello, C. (1985). Hemisphere dynamics in lexical access: Automatic and controlled priming. Brain \& Language, 26, 146-172.

COULL, J. T., \& NoBre, A. C. (1998). Where and when to pay attention: The neural systems for directing attention to spatial locations and to time intervals as revealed by both PET and fMRI. Journal of Neuroscience, 18, 7426-7435.

Delis, D. C., Robertson, L. C., \& Efron, R. (1986). Hemispheric specialization of memory for visual hierarchical stimuli. Neuropsychologia, 24, 205-214.

Deubel, H., \& Schneider, W. X. (1996). Saccade target selection and object recognition: Evidence for a common attentional mechanism. Vision Research, 36, 1827-1837.

Dickinson, C. A., \& Intraub, H. (2008). Transsaccadic representation of layout: What is the time course of boundary extension? Journal of Experimental Psychology: Human Perception \& Performance, 34, 543-555.

Ebersbach, G., Trottenberg, T., Hättig, H., Schelosky, L., Schrag, A., \& Poewe, W. (1996). Directional bias of initial visual exploration: A symptom of neglect in Parkinson's disease. Brain, 119, 79-87.

Ellis, A. W., Young, A. W., \& Anderson, C. (1988). Modes of visual word recognition in the left and right cerebral hemispheres. Brain \& Language, 35, 254-273. 
Epstein, R. A. (2005). The cortical basis of visual scene processing. Visual Cognition, 12, 954-978.

Faust, M., Kravetz, S., \& BabKoff, H. (1993). Hemispheric specialization or reading habits: Evidence from lexical decision research with Hebrew words and sentences. Brain \& Language, 44, 254-263.

Fecteau, J. H., \& Enns, J. T. (2005). Visual letter matching: Hemispheric functioning or scanning biases? Neuropsychologia, 43, 14121428.

Friedman, A. (1979). Framing pictures: The role of knowledge in automatized encoding and memory for gist. Journal of Experimental Psychology: General, 108, 316-355.

Gitelman, D. R., Nobre, A. C., Parrish, T. B., LaBar, K. S., Kim, Y.-H., Meyer, J. R., \& Mesulam, M.-M. (1999). A large-scale distributed network for covert spatial attention: Further anatomical delineation based on stringent behavioural and cognitive controls. Brain, 122, 1093-1106.

Göbel, S. M., Calabria, M., Farnè, M., \& Rossetti, Y. (2006). Parietal rTMS distorts the mental number line: Simulating "spatial" neglect in healthy subjects. Neuropsychologia, 44, 860-868.

Gottesman, C. V., \& Intraub, H. (2002). Surface construal and the mental representation of scenes. Journal of Experimental Psychology: Human Perception \& Performance, 28, 589-599.

Hausmann, M., Corballis, M. C., \& Fabri, M. (2003). Line bisection in the split brain. Neuropsychology, 17, 602-609.

Heilman, K. M., Bowers, D., Valenstein, E., \& Watson, R. T. (1987). Hemispace and hemispatial neglect. In M. Jeannerod (Ed.), Neurophysiological and neuropsychological aspects of spatial neglect (pp. 115-150). Amsterdam: Elsevier.

Heilman, K. M., \& Valenstein, E. (1972). Auditory neglect in man. Archives of Neurology, 26, 32-35.

Heilman, K. M., \& Valenstein, E. (1985). Clinical neuropsychology. New York: Oxford University Press.

Henderson, J. M., Brockmole, J. R., Castelhano, M. S., \& Mack, M. (2007). Visual saliency does not account for eye movements during visual search in real-world scenes. In R. P. G. van Gompel, M. H. Fischer, \& R. L. Hill (Eds.), Eye movements: A window on mind and brain (pp. 537-562). Amsterdam: Elsevier.

Henderson, J. M., \& Hollingworth, A. (1999). The role of fixation position in detecting scene changes across saccades. Psychological Science, 10, 438-443.

Hines, D. (1978). Visual information processing in the left and right hemispheres. Neuropsychologia, 16, 593-600.

Hoffman, J. E., \& Subramaniam, B. (1995). The role of visual attention in saccadic eye movements. Perception \& Psychophysics, 57, 787-795.

Hollingworth, A., \& Henderson, J. M. (2002). Accurate visual memory for previously attended objects in natural scenes. Journal of Experimental Psychology: Human Perception \& Performance, 28, 113-136.

INTRAUB, H. (2004). Anticipatory spatial representation of 3D regions explored by sighted observers and a deaf-and-blind observer. Cognition, 94, 19-37.

InTRAUB, H. (2007). Scene perception: The world through a window. In M. A. Peterson, B. Gillam, \& H. A. Sedgwick (Eds.), Mental structure in visual perception: Julian Hochberg's contributions to our understanding of the perception of pictures, film, and the world (pp. 454466). New York: Oxford University Press.

Intraub, H., \& Bodamer, J. L. (1993). Boundary extension: Fundamental aspect of pictorial representation or encoding artifact? Journal of Experimental Psychology: Learning, Memory, \& Cognition, 19, 1387-1397.

Intraub, H., Daniels, K. K., Horowitz, T. S., \& Wolfe, J. M. (2008). Looking at scenes while searching for numbers: Dividing attention multiplies space. Perception \& Psychophysics, 70, 1337-1349.

Intraub, H., \& Dickinson, C. A. (2008). False memory 1/20th of a second later: What the early onset of boundary extension reveals about perception. Psychological Science, 19, 1007-1014.

Intraub, H., Gottesman, C. V., \& Bills, A. J. (1998). Effects of perceiving and imagining scenes on memory for pictures. Journal of Experimental Psychology: Learning, Memory, \& Cognition, 24, 186-201.
Intraub, H., Hoffman, J. E., Wetherhold, C. J., \& Stoehs, S.-A. (2006). More than meets the eye: The effect of planned fixations on scene representation. Perception \& Psychophysics, 68, 759-769.

INTRAUB, H., \& RiCHARDSON, M. (1989). Wide-angle memories of closeup scenes. Journal of Experimental Psychology: Learning, Memory, \& Cognition, 15, 179-187.

Irwin, D. E., \& Zelinsky, G. J. (2002). Eye movements and scene perception: Memory for things observed. Perception \& Psychophysics, 64, 882-895.

ITTI, L., \& KосH, C. (2000). A saliency-based search mechanism for overt and covert shifts of visual attention. Vision Research, 40, 1489-1506.

IтTI, L., \& KoсH, C. (2001). Computational modeling of visual attention. Nature Reviews Neuroscience, 2, 194-203.

Jewell, G., \& McCourt, M. E. (2000). Pseudoneglect: A review and meta-analysis of performance factors in line bisection tasks. Neuropsychologia, 38, 93-110.

Karnath, H. O., Milner, A. D., \& Vallar, G. (Eds.) (2002). The cognitive and neural bases of spatial neglect. New York: Oxford University Press.

Kinsbourne, M. (1970). The cerebral basis of lateral asymmetries in attention. Acta Psychologica, 33, 193-201.

Kowler, E., Anderson, E., Dosher, B., \& Blaser, E. (1995). The role of attention in the programming of saccades. Vision Research, 35, 1897-1916.

LEIBER, L. (1976). Lexical decisions in the left and right cerebral hemispheres. Brain \& Language, 3, 443-450.

LiNDELL, A. K. (2006). In your right mind: Right hemisphere contributions to language processing and production. Neuropsychology Review, 16, 131-148.

Loftus, G. R., \& Mackworth, N. H. (1978). Cognitive determinants of fixation location during picture viewing. Journal of Experimental Psychology: Human Perception \& Performance, 4, 565-572.

Luh, K. E., Rueckert, L. M., \& Levy, J. (1991). Perceptual asymmetries for free viewing of several types of chimeric stimuli. Brain \& Cognition, 16, 83-103.

MarTin, M. (1979). Hemispheric specialization for local and global processing. Neuropsychologia, 17, 33-40.

Mattingley, J. B., Berberovic, N., Corben, L., Slavin, M. J., NichOLls, M. E. R., \& BRAdShaw, J. L. (2004). The greyscales task: A perceptual measure of attentional bias following unilateral hemispheric damage. Neuropsychologia, 42, 387-394.

MCCourT, M. E. (2001). Performance consistency of normal observers in forced-choice tachistoscopic visual line bisection. Neuropsychologia, 39, 1065-1076.

McCourt, M. E., Garlinghouse, M., \& Reuter-Lorenz, P. A. (2005). Unilateral visual cueing and asymmetric line geometry share a common attentional origin in the modulation of pseudoneglect. Cortex, 41, 499-511.

McCourt, M. E., \& Jewell, G. (1999). Visuospatial attention in line bisection: Stimulus modulation of pseudoneglect. Neuropsychologia, 37, 843-855.

Mesulam, M.-M. (1981). A cortical network for directed attention and unilateral neglect. Annals of Neurology, 10, 309-325.

MishKIN, M., \& Forgays, D. G. (1952). Word recognition as a function of retinal locus. Journal of Experimental Psychology, 43, 43-48.

Morikawa, K., \& McBeath, M. K. (1992). Lateral motion bias associated with reading direction. Vision Research, 32, 1137-1141.

Nicholls, M. E. R., Bradshaw, J. L., \& MatTingley, J. B. (1999). Free-viewing perceptual asymmetries for the judgement of brightness, numerosity and size. Neuropsychologia, 37, 307-314.

Nicholls, M. E. R., \& Loftus, A. M. (2007). Pseudoneglect and neglect for mental alphabet lines. Brain Research, 1152, 130-138.

Nicholls, M. E. R., \& RoberTs, G. R. (2002). Can free-viewing perceptual asymmetries be explained by scanning, pre-motor or attentional biases? Cortex, 38, 113-136.

Nobre, A. C., Coull, J. T., Frith, C. D., \& Mesulam, M. M. (1999). Orbitofrontal cortex is activated during breaches of expectation in tasks of visual attention. Nature Neuroscience, 2, 11-12.

Park, S. J., Intraub, H., Yi, D.-J., Widders, D., \& Chun, M. M. (2007). Beyond the edges of a view: Boundary extension in human scene-selective visual cortex. Neuron, 54, 335-342. 
Parkhurst, D., Law, K., \& Niebur, E. (2002). Modeling the role of salience in the allocation of overt visual attention. Vision Research, 42, 107-123.

Pollack, I. (1970). A nonparametric procedure for evaluation of true and false positives. Behavior Research Methods \& Instrumentation, 2, 155-156.

Pollack, I., \& Norman, D. A. (1964). A nonparametric analysis of recognition experiments. Psychonomic Science, 1, 125-126.

Pollatsek, A., Bolozky, S., Well, A. D., \& Rayner, K. (1981). Asymmetries in the perceptual span for Israeli readers. Brain \& Language, 14, 174-180.

RAYNER, K. (1998). Eye movements in reading and information processing: 20 years of research. Psychological Bulletin, 124, 372-422.

Schmidt, G. L., DeBuse, C. J., \& Seger, C. A. (2007). Right hemisphere metaphor processing? Characterizing the lateralization of semantic processes. Brain \& Language, 100, 127-141.

Shepherd, M., Findlay, J. M., \& Hockey, R. J. (1986). The relationship between eye movements and spatial attention. Quarterly Journal of Experimental Psychology, 38A, 475-491.

Siman-Tov, T., Mendelsohn, A., Schonberg, T., Avidan, G., PodLIPSKY, I., PESSOA, L., ET AL. (2007). Bihemispheric leftward bias in a visuospatial attention-related network. Journal of Neuroscience, 27, 11271-11278.

Snodgrass, J. G., Volvovitz, R., \& Walfish, E. R. (1972). Recognition memory for words, pictures, and words + pictures. Psychonomic Science, 27, 345-347.
SpaleK, T. M., \& Hammad, S. (2005). The left-to-right bias in inhibition of return is due to the direction of reading. Psychological Science, 16, 15-18.

Varnava, A., \& Halligan, P. W. (2007). Influence of age and sex on line bisection: A study of normal performance with implications for visuospatial neglect. Aging, Neuropsychology, \& Cognition, 14, 571-585.

VOLBERG, G., \& HüBNER, R. (2004). On the role of response conflicts and stimulus position for hemispheric differences in global/local processing: An ERP study. Neuropsychologia, 42, 1805-1813.

Weintraub, S., \& Mesulam, M.-M. (1987). Right cerebral dominance in spatial attention: Further evidence based on ipsilateral neglect. $\mathrm{Ar}$ chives of Neurology, 44, 621-625.

Williams, D. E., \& Reingold, E. M. (2001). Preattentive guidance of eye movements during triple conjunction search tasks: The effects of feature discriminability and saccadic amplitude. Psychonomic Bulletin \& Review, 8, 476-488.

Young, A. W., \& Ellis, A. W. (1985). Different methods of lexical access for words presented in the left and right visual hemifields. Brain \& Language, 24, 326-358.

ZELINSKY, G. J. (1996). Using eye saccades to assess the selectivity of search movements. Vision Research, 36, 2177-2187.

(Manuscript received June 27, 2008; revision accepted for publication March 11, 2009.) 